\title{
The changing treatment of metastatic her2-positive breast cancer (Review)
}

\author{
MARIA MITSOGIANNI, IOANNIS P. TRONTZAS, GEORGIA GOMATOU, \\ STEPHANIE IOANNOU, NIKOLAOS K. SYRIGOS and ELIAS A. KOTTEAS
}

Oncology Unit, 'Sotiria' General Hospital, Athens School of Medicine, Athens 11527, Greece

Received November 13, 2020; Accepted January 19, 2021

DOI: 10.3892/ol.2021.12548

\begin{abstract}
Human epidermal growth factor receptor 2 (HER2)-overexpressing breast cancer has been historically associated with an aggressive disease course with common distant metastasis and poor prognosis. HER2-targeting therapies have significantly changed treatment and drastically improved outcomes for this group of patients. However, primary or acquired resistance to anti-HER2 regimens leads almost universally to disease progression, often with difficult to treat central nervous system (CNS) metastases. The current review summarized the existing therapeutic options for HER2-positive metastatic disease in the first, second and further line setting. Furthermore, novel agents currently under development were presented, which have demonstrated encouraging results in heavily pretreated patients or specific subgroups, such as HR-positive/HER2-positive tumors and CNS disease.
\end{abstract}

Correspondence to: Dr Maria Mitsogianni, Oncology Unit, 'Sotiria' General Hospital, Athens School of Medicine, Mesogion 152, Athens 11527, Greece

E-mail: mitsogiannimaria@gmail.com

Abbreviations: ADCC, antibody-dependent cellular cytotoxicity; CDK, cyclin dependent kinase; CNS, central nervous system; CTLA4, cytotoxic T-lymphocyte antigen 4; DCR, disease control rate; EGFR, epidermal growth factor receptor; ER, estrogen receptor; FcR, fc-receptor; FDA, Food and Drug Administration; HER2, human epidermal growth factor receptor 2; HR, hormone receptor; MAPK, mitogen-activated protein kinase; mTOR, mechanistic target of rapamycin; NK, natural killer cells; ORR, objective response rate; OS, overall survival; $\mathrm{PD}-1$, programmed cell death protein 1; PD-L1, programmed death-ligand 1; PFS, progression free survival; PI3K, phosphatidylinositol-4,5-biphosphate 3-kinase; T-DM1, ado Trastuzumab emtasine; TILs, tumor-infiltrating lymphocytes; TKI, tyrosine kinase inhibitor; TMB, tumor mutational burden; TTP, time to progression

Key words: metastatic breast cancer, HER2-positive, HER2-targeting agents, triple-positive breast cancer, immunotherapy

\author{
Contents \\ 1. Introduction \\ 2. Established HER2-targeting agents \\ 3. Triple positive breast cancer \\ 4. Novel agents \\ 5. Proposed treatment algorithm \\ 6. Conclusion
}

\section{Introduction}

Breast cancer is the most common malignancy and first cause of cancer-related death among women worldwide (1). In the last years it has become clear that breast cancer is a heterogeneous disease with distinct molecular characteristics and clinical behavior. Human epidermal growth factor receptor 2 (HER2) gene amplification resulting in overexpression of the HER 2 protein can be detected in approximately $15-20 \%$ of invasive breast tumors and is, historically, associated with an aggressive disease course with common distant metastases and poor prognosis (2-4).

HER2, a member of the epidermal growth factor family, is a transmembrane protein consisting of an extracellular ligand-binding domain, a transmembrane domain and an intracellular domain with tyrosine kinase activity. Homo- and heterodimerization of the HER family proteins [epidermal growth factor receptor (EGFR), HER2, HER3, HER4], mostly involving HER2 as the preferred dimerization partner, leads to activation of various downstream signaling pathways such as mitogen-activated protein kinase (MAPK) and phosphatidylinositol-4,5-biphosphate 3-kinase (PI3K) that promote cell proliferation, migration, survival and invasion $(5,6)$.

The introduction of therapies inhibiting the HER 2 signaling has drastically altered the landscape of treatment with significantly improved outcomes in this group of patients (7). However, primary or acquired resistance to anti-HER 2 regimens leads almost universally to disease progression, often with development of difficult to treat central nervous system (CNS) metastases. In this review, we will summarize the existing therapeutic options for HER2-positive metastatic disease as well as novel agents currently under development that are likely to further improve prognosis. 


\section{Established HER2-targeting agents}

Trastuzumab. Trastuzumab is a recombinant humanized IgG monoclonal antibody directed against the extracellular domain of HER2 with various molecular mechanisms (8). Upon binding to its receptor, trastuzumab induces internalization and degradation of HER2, inhibits the HER2-dependent downstream proliferation signaling and activates natural killer (NK) cells in the tumor microenvironment through antibody-dependent cellular cytotoxicity (ADCC). Trastuzumab was the first targeted agent to demonstrate impressive efficacy as monotherapy as well as in combination with chemotherapy in the first-line setting and the first monoclonal antibody to be approved for the treatment of metastatic HER2-positive breast cancer in $1998(9,10)$.

Trastuzumab and chemotherapy in the first line setting. According to the landmark study of Slamon et al (10), the addition of trastuzumab to taxane or anthracycline/cyclophosphamide resulted in higher objective response rate (ORR) (50 vs. $32 \%, \mathrm{P}<0.001$ ), longer progression free survival (PFS) (7.4 vs. 4.6 months, $\mathrm{HR}=0.5,95 \% \mathrm{CI}$ : $0.41-0.63, \mathrm{P}<0.001$ ) and overall survival (OS) (25.1 vs. 20.3 months, $\mathrm{HR}=0.8,95 \%$ CI: 0.64-1.00, $\mathrm{P}=0.046)$ compared to chemotherapy alone in patients with HER2-positive metastatic breast cancer (10). Because of the cardiotoxicity attributed to the combination of trastuzumab with anthracycline (cardiac dysfunction in 27 vs. $13 \%$ of the patients), trastuzumab and taxane was since adopted as the preferred treatment.

It has been suggested that vinorelbine could be an alternative option for taxanes in this setting, demonstrating similar PFS (12.4 vs. 15.3 months HR=0.94, 95\% CI: 0.71-1.25; $\mathrm{P}=0.67$ ) and $\mathrm{OS}$ rates (35.7 vs. 38.8 months, $\mathrm{HR}=1.01,95 \%$ CI: $0.71-1.42$; $\mathrm{P}=0.98$ ) in a phase III trial (11). On the other hand, triple therapy with the addition of carboplatin $(12,13)$ or non-pegylated liposomal doxorubicin (14) to trastuzumab and taxane showed no OS benefit and was associated with excessive toxicity.

Sequential trastuzumab and chemotherapy. Sequential treatment with initial single-agent trastuzumab followed by the addition of chemotherapy upon progression has always been considered an appealing strategy, given that it delays the initiation of chemotherapy. It has been evaluated in three randomized trials which, however, failed to demonstrate non-inferiority.

In the phase II HERTAX trial (15), patients received either trastuzumab monotherapy and switched to docetaxel at progression or upfront trastuzumab and docetaxel combination. ORR was better in the combination group (79 vs. $53 \%, \mathrm{P}=0.016$ ) and, despite similar PFS in both groups (9.4 vs. 9.9 months, $\mathrm{HR}=1.33$, 95\% CI: $0.86-2.06, \mathrm{P}=0.2$ ), OS was longer in the combination arm, with a trend towards statistical significance (30.5 vs. 19.7 months, $\mathrm{HR}=1.49,95 \%$ CI: 0.91-2.47, $\mathrm{P}=0.12$ ).

The phase III JO17360 trial (16) evaluated single-agent trastuzumab followed by the addition of docetaxel after progression vs. upfront docetaxel and trastuzumab combination. PFS after both trastuzumab and docetaxel was comparable (12.4 vs. 14.6 months, $\mathrm{HR}=1.35,95 \% \mathrm{CI}$ : $0.79-2.30$, $\mathrm{p}=0.27$ ). Nevertheless, patient accrual was terminated prematurely due to better PFS in the combination arm compared to trastuzumab monotherapy (14.6 vs. 3.7 months, $\mathrm{HR}=4.24,95 \%$ CI:
2.48-7.24, $\mathrm{P}<0.01$ ), which was the primary endpoint of the study. OS was also significantly better in the upfront combination arm (median OS not available due to small number of deaths, $\mathrm{HR}=2.72,95 \%$ CI: $1.03-7.18, \mathrm{P}=0.04)$.

SAKK 22/99 (17), a phase III study with similar design to JO17360, randomized patients to receive either trastuzumab followed by trastuzumab plus chemotherapy or upfront trastuzumab plus chemotherapy. Time to progression (TTP) and OS did not differ significantly between the two groups (12.2 vs. 10.3 months, $\mathrm{HR}=0.7,95 \% \mathrm{CI}: 0.5-1.1, \mathrm{P}=0.10$ and 35.6 vs. 36.3 months, $\mathrm{HR}=0.9, \mathrm{P}=0.55$, respectively). In subgroup analysis of the trastuzumab alone arm, patients without visceral disease had a significantly longer TTP compared to the ones with visceral metastases (21.8 vs. 10.1 months, $\mathrm{P}=0.03$ ). Based on these findings, the authors suggest that single-agent trastuzumab could be a sufficient treatment option for individual patients without visceral involvement.

Pertuzumab. Like trastuzumab, pertuzumab is a recombinant monoclonal antibody against HER2. It binds to a different extracellular dimerization domain than trastuzumab and prevents HER 2/HER3 heterodimerization, thereby acting complementarily to trastuzumab in inhibiting the downstream proliferation pathways (6).

Pertuzumab, trastuzumab and docetaxel in the first-line setting. Dual HER2 inhibition with trastuzumab and pertuzumab in combination with chemotherapy has been introduced as the current standard-of-care in first-line treatment based on the results of the phase III CLEOPATRA study $(18,19)$. Previously untreated patients were randomized to receive trastuzumab and docetaxel with or without pertuzumab. The addition of pertuzumab led to significant improvement of ORR ( 80 vs. $69 \%, \mathrm{P}=0.001$ ), $\mathrm{PFS}$ (18.5 vs. 12.4 months, $\mathrm{HR}=0.62$, 95\% CI: $0.51-0.75, \mathrm{P}<0.001)$ and OS (56.5 vs. 40.8 months, HR $=0.68$; 95\% CI: $0.56-0.84, \mathrm{P}<0.001)$. Recently, the end-of-study analysis further confirmed the durable superiority of the dual blockade with 8-year OS rates of 37 and 23\%, respectively (20). According to a post-hoc analysis, continuing the administration of docetaxel after 6 cycles did not add clinical benefit in terms of PFS or OS (21). Patients who had previously received trastuzumab in the neoadjuvant or adjuvant setting were also found to experience similar benefit from the three-agent combination.

Pertuzumab, trastuzumab and other chemotherapy-regimens in the first-line setting. Chemotherapy regimens other than docetaxel have been evaluated in combination with trastuzumab and pertuzumab. As shown in the preliminary results of the PERUSE trial (22), paclitaxel-containing regimens are valid alternative options to docetaxel. ORR was $79 \%$ with docetaxel, $83 \%$ with paclitaxel and $77 \%$ with nab-paclitaxel, while PFS was 19.6, 23.0 and 18.1 months, respectively. Toxicity was in accordance with known safety profiles, with docetaxel being associated more frequently with febrile neutropenia (11 vs. $1 \%$ ) and mucositis (25 vs. $14 \%$ ) and less frequently with peripheral neuropathy (16 vs. $31 \%$ ). Smaller single-arm studies have demonstrated efficacy for vinorelbine (ORR 74,2\%, PFS 14.3 months) (23) and eribulin (ORR 80\%) combined with dual HER2 blockade (24). 
Pertuzumab, trastuzumab and chemotherapy in the second-line setting. Efficacy of dual HER2-blockade with trastuzumab and pertuzumab in patients previously treated with trastuzumab for advanced disease was investigated in the phase III PHEREXA trial (25). Patients who had progressed on trastuzumab and taxane in the first-line setting were randomized to receive trastuzumab and capecitabine with or without the addition of pertuzumab. The primary endpoint of PFS (11.1 vs. 9 months, HR=0.82, 95\% CI: 0.65-1.02, $\mathrm{P}=0.07$ ) was not reached, although there was a non-significant improvement in the pertuzumab arm. Similarly, there was a numerical survival benefit in the pertuzumab group (OS 36.1 vs. 28.1 months, HR=0.76; 95\% CI: 0.60-0.98), but statistical significance was not claimed as the trial was underpowered for OS analysis (26). Although the combination of trastuzumab and pertuzumab does seem to offer clinical benefit beyond the first-line setting and the reported median PFS is comparable to the one demonstrated with ado trastuzumab emtasine (T-DM1), PHEREXA is a negative trial and T-DM1 remains the current standard-of-care in the second-line setting.

Ado Trastuzumab emtasine (T-DM1). T-DM1 is an antibody-drug conjugate composed of trastuzumab and the potent microtubule-inhibitory agent DM1. In binding HER2, the cytotoxic component is selectively delivered to HER $2^{+}$cells and leads to apoptosis upon intracellular release (27).

T-DM1 in the second-and third-line setting. Efficacy of T-DM1 as third- and second-line treatment was demonstrated in TH3RESA $(28,29)$ and EMILIA $(30,31)$ trials, respectively. TH3RESA compared T-DM1 to treatment of physician's choice after previous exposure to trastuzumab, lapatinib and taxane and demonstrated a significantly longer PFS (6.2 vs. 3.3 months, $\mathrm{HR}=0.53$, 95\% CI: 0.42-0.66, $\mathrm{P}<0.0001$ ) and $\mathrm{OS}$ (22.7 vs. 15.8 months, $\mathrm{HR}=0.68,95 \% \mathrm{CI}: 0.54-0.85$, $\mathrm{P}=0.0007)$ in the T-DM1 group, thereby establishing T-DM1 as preferred therapy in the third line. T-DM1 was also associated with a lower incidence of grade 3 or worse adverse events (32 vs. $43 \%$ ).

In EMILIA, patients previously treated with trastuzumab and a taxane were randomized to receive T-DM1 or lapatinib plus capecitabine-the standard-of-care at the time. T-DM1 was associated with significantly improved PFS (9.6 vs. 6.4 months, $\mathrm{HR}=0.65,95 \% \mathrm{CI}$ : $0.55-0.77, \mathrm{P}<0.001)$ and $\mathrm{OS}$ (30.9 vs. 25.1 months, $\mathrm{HR}=0.68,95 \% \mathrm{CI}: 0.55-0.85, \mathrm{P}<0.001$ ) and lower toxicity (grade $3 / 4$ adverse events in 41 vs. $57 \%$ of the patients). Based on these results, T-DM1 was rapidly adopted as the preferred regimen in the second line.

T-DM1 in the first-line setting. T-DM1 in the first-line was evaluated in the MARIANNE trial $(32,33)$. In this randomized phase III study, previously untreated patients were divided in three treatment arms: T-DM1, T-DM1 plus pertuzumab and trastuzumab plus taxane. The study demonstrated non-inferiority-but also no superiority - concerning the primary endpoint of PFS for both the T-DM1 and T-DM1 plus pertuzumab arms (14.1 and 15.2 months, respectively vs. 13.7 months in the trastuzumab plus taxane group, $\mathrm{P}=0.31$ and 0.14). OS exceeded 50 months and was also similar across three treatment arms. T-DM1-containing arms were associ- ated with lower treatment discontinuation rates due to adverse events and better quality of life. However, since comparison was not made to a group receiving dual HER2 blockade, which is the current standard-of-care, T-DM1 remains an alternative first-line option mainly for patients unsuitable to receive the preferred regimen.

Lapatinib. Lapatinib is a small-molecule dual tyrosine kinase inhibitor (TKI) reversibly targeting the intracellular domain of both EGFR and HER2 and blocking activation of downstream proliferation pathways and was, until recently, the only TKI approved in the treatment of HER2 ${ }^{+}$breast cancer (34).

Historically, lapatinib in combination with capecitabine was a commonly used treatment upon progression on trastuzumab plus chemotherapy (35). However, since superiority of T-DM1 and, more recently, neratinib was demonstrated, this regimen is reserved for later lines of therapy.

Lapatinib in the first-line setting. Lapatinib is inferior to trastuzumab in the first-line setting, as shown in the MA.31 trial (36), in which previously untreated patients with metastatic disease received taxane and HER2 blockade with either trastuzumab or lapatinib. Lapatinib was associated with shorter intention-to-treat PFS (9.0 vs. 11.3 months, $\mathrm{HR}=1.37$, 95\% CI: $1.13-1.65, \mathrm{P}=0.001$ ), which was the primary endpoint of the study. Toxicity was also higher in the lapatinib arm, with more incidents of grade $3 / 4$ diarrhea and rash $(\mathrm{P}<0.001)$.

Lapatinib +/- trastuzumab in pretreated patients. According to the findings of the EFG104900 trial (37), combination of lapatinib and trastuzumab in trastuzumab-exposed patients is superior to lapatinib alone in terms of PFS (11.1 vs. 8.1 weeks, $\mathrm{HR}=0.74,95 \% \mathrm{CI}$ : $0.58-0.94, \mathrm{P}=0.011)$ and $\mathrm{OS}$ (14 vs. 9.5 months, $\mathrm{HR}=0.74,95 \%$ CI: $0.57-0.97, \mathrm{P}=0.026$ ), with the exception of hormone receptor (HR)-positive tumors, which are known to demonstrate higher levels of HER2 resistance (38). Therefore, combination of lapatinib plus trastuzumab can be considered a chemotherapy-free alternative for heavily pretreated patients.

\section{Triple positive breast cancer}

Approximately $50 \%$ of HER2-positive breast tumors also express HR (38). There is a known bidirectional crosstalk between the estrogen receptor (ER) and HER receptor families, resulting in overexpression of ER in the presence of acquired HER 2 resistance and resistance to hormonal treatment through HER 2 overexpression (39). The obvious therapeutic strategy for this group of patients includes simultaneous blocking of both signaling pathways with a combination of HER2-targeted agents and endocrine therapy. It has been previously shown that addition of trastuzumab or lapatinib to an aromatase inhibitor prolongs PFS, although not OS, compared to endocrine therapy alone in the metastatic setting (40-42). Addition of endocrine therapy to HER2-targeted treatment is much less studied although widely used - there is to date no randomized trial.

Historically, clinical trials did not distinguish the HR status in HER2 ${ }^{+}$disease. Dual HER blockade plus chemotherapy has been the standard of care for these patients, although the idea 
of chemotherapy-free regimens has always been appealing. Efficacy of endocrine treatment combined with single-agent or dual HER 2 blockade in the absence of chemotherapy has been recently investigated in the PERTAIN and ALTERNATIVE trials $(43,44)$.

In the phase II PERTAIN trial, treatment-naïve patients (with exception of endocrine therapy) were randomized to receive trastuzumab and an aromatase inhibitor with or without Pertuzumab (43). The primary endpoint of the study was PFS, which was shown to be significantly better with the dual blockade (18.9 vs. 15.8 months, HR=0.65, 95\% CI: 0.48-0.89, $\mathrm{P}=0.007$ ). ORR was similar between the two arms ( 63.3 vs. $55.7 \%, \mathrm{P}=0.254$ ), nevertheless, duration of response was significantly longer in the pertuzumab group (27.1 vs. 15.1 months, $\mathrm{HR}=0.57,95 \%$ CI: $0.36-0.91, \mathrm{P}=0.018$ ).

ALTERNATIVE (44), a randomized, phase III trial, evaluated first-line treatment with an aromatase inhibitor combined with trastuzumab or lapatinib or both in postmenopausal women. Patients in the dual blockade arm had a significantly longer PFS compared to trastuzumab (11.0 vs. 5.7 months, HR $=0.62,95 \%$ CI: $0.45-0.88, P=0.0064$ ), which was the primary endpoint of the study. Median PFS with lapatinib was 8.3 months (HR vs. trastuzumab $0.71,95 \%$ CI: 0.51-0.98, $\mathrm{P}=0.036$ ). Adverse events like diarrhea, rash and paronychia were more common in the lapatinib containing regimens, mostly grade 1 and 2 .

Both trials suggest encouraging efficacy of dual HER2 blockade and endocrine therapy in previously untreated HER2-positive, HR-positive disease, thereby offering an alternative chemotherapy-free option for selected patients unfit to receive chemotherapy.

\section{Novel agents}

New generation TKIs. Novel TKIs currently adopted in clinical practice include neratinib and pyrotinib, two irreversible pan-HER TKIs targeting EGFR, HER2 and HER4, as well as tucatinib, a highly selective HER2 inhibitor with minimal inhibition of EGFR, therefore with decreased potential for EGFR-related toxicities.

Neratinib in later-line setting. The phase III NALA trial (45) compared neratinib plus capecitabine to lapatinib plus capecitabine after two or more anti-HER2 containing treatment lines. Treatment with neratinib resulted in significantly longer PFS (8.8. vs. 6.6 months, $\mathrm{P}=0.0003)$ and a trend towards improved OS (24.0 vs. 22.2 months, $\mathrm{HR}=0.88,95 \% \mathrm{CI}$ : $0.72-1.07, \mathrm{P}=0.2086$ ). Grade 3 diarrhea was more common in the neratinib arm (24 vs. 13\%), although discontinuation of treatment due to adverse events was lower with neratinib (10.9 vs. $14.5 \%$ ). Based on these findings, the combination of neratinib and capecitabine has been approved from the Food and Drug Administration (FDA) in April 2020 as third-line treatment in metastatic HER2-positive breast cancer.

Neratinib in the first-line setting. In the phase II NEfERT-T trial (46), neratinib failed to demonstrate superiority against trastuzumab as first-line therapy. Patients with previously untreated advanced disease were randomized to receive paclitaxel with either trastuzumab or neratinib. There was no difference in the median PFS (12.9 months in both arms), while neratinib was associated with higher incidence of grade 3 diarrhea (30.4 vs. $3.8 \%)$. However, a sub-analysis of NEfERT-T showed neratinib to be more effective than trastuzumab in preventing CNS disease (relative risk of brain metastases 0.48 , $\mathrm{P}=0.002$ ).

Neratinib in CNS disease. CNS activity of neratinib has also been tested outside the NEfERT-T trial. In one cohort of the single-arm multi-cohort TBCRC 022 trial (47), forty patients with progressive CNS disease after at least one line of CNS-directed treatment were treated with neratinib monotherapy. ORR was only $8 \%$, resulting in a median PFS of 1.9 months. However, combination of neratinib and capecitabine in another cohort of the same trial (48) resulted in more promising outcomes. Patients with or without prior exposure to lapatinib were treated with neratinib and capecitabine, with an ORR of 33 and 49\%, PFS of 3.1 and 5.5 months and OS of 15.1 and 13.3 months, respectively. As noted in the study, these findings support a synergy between HER2-targeted agents and chemotherapy.

Pyrotinib in second-line setting. Efficacy of pyrotinib has been recently evaluated in a Chinese open-label, randomized phase II study (49). Patients enrolled in the study had been previously treated with chemotherapy and trastuzumab and were randomized to receive either pyrotinib or lapatinib - both drugs in combination with capecitabine. ORR was the primary endpoint of the study and was significantly better in the pyrotinib group (78.5 vs. $57.1 \%$, treatment difference $21.3 \%$, 95\% CI: 4.0-38.7, $\mathrm{P}=0.01)$. Pyrotinib demonstrated significant superiority in terms of PFS as well (18.1 vs. 7.0 months, $\mathrm{HR}=0.36,95 \%$ CI: $0.23-0.58, \mathrm{P}<0.001)$. As far as toxicity is concerned, grade $3 / 4$ hand-foot syndrome and diarrhea were more common in the pyrotinib arm (24.6 vs. $20.6 \%$ and 15.4 vs. $4.8 \%$, respectively). These findings were confirmed in the phase III PHOEBE trial (50) comparing pyrotinib plus capecitabine to lapatinib plus capecitabine after exposure to trastuzumab and taxane. PFS was significantly longer with pyrotinib (12.5 vs. 6.8 months, HR=0.39, 95\% CI: 0.27-0.56, $\mathrm{P}<0.0001$ ), while the toxicity profile was in accordance with the one of the phase II trial.

Another randomized phase $\approx$ III trial (51) evaluated capecitabine with pyrotinib or placebo in Chinese patients after failure of trastuzumab and taxane. PFS was significantly longer in the pyrotinib arm (11.1 vs. 4.1 months). According to the study design, patients who progressed on placebo plus capecitabine received pyrotinib monotherapy. In this subgroup of patients, ORR was $38 \%$ and PFS 5.5 months. The most common adverse event in the pyrotinib arm was grade 3 diarrhea, which was observed in $30.8 \%$ of patients.

The biggest limitation of these trials is that, among the study population, there was no prior exposure to current standards of care in earlier lines such as pertuzumab and T-DM1. However, pyrotinib in combination with capecitabine has already received approval in China for HER $2^{+}$breast cancer after anthracycline or taxane chemotherapy (52).

Tucatinib in later-line setting. Tucatinib was recently granted Breakthrough Therapy designation by the FDA in combina- 
tion with trastuzumab and capecitabine as second or later line treatment, based on the positive results of the HER2CLIMB trial (53). In this randomized trial, patients pretreated with trastuzumab, pertuzumab and T-DM1 received a combination of trastuzumab and capecitabine with or without tucatinib. The addition of tucatinib resulted in improved PFS at 1 year (33.1 vs. $12.3 \%$, HR for progression or death $0.54,95 \%$ CI: $0.42-0.71$, $\mathrm{P}<0.001)$. ORR (40.6 vs. $22.8 \%$ ) and OS at 2 years $(44.9$ vs. $26.6 \%$, HR for death $0.66,95 \% \mathrm{CI}$ : $0.50-0.88, \mathrm{P}=0.005)$ were significantly better in the tucatinib group as well.

Tucatinib in CNS disease. Interestingly, patients with brain metastases were included in HER2CLIMB, composing almost half of the study population $(47.5 \%)$, and seemed to benefit particularly from the triplet therapy (PFS at 1 year 24.9 vs. $0 \%$, $\mathrm{HR}=0.48$, 95\% CI: 0.34-0.69, P<0.001, median CNS-PFS 9.9 vs. 4.2 months, median $\mathrm{OS} 18.1$ vs. 12.0 months, $\mathrm{HR}=0.58$, 95\% CI: $0.40-0.85, \mathrm{P}=0.005$ ) (54). Since brain metastases appear often in the course of the disease, these findings make tucatinib an extremely promising later-line option.

Antibody-drug conjugate Trastuzumab deruxtecan (DS-8201). Trastuzumab deruxtecan is an antibody-drug conjugate comprised of an anti-HER2 antibody bound to a cytotoxic topoisomerase I inhibitor (a derivative of irinotecan) (55). It has been recently granted accelerated approval by the FDA for patients who have received at least two anti-HER2-based treatment lines. Efficacy was evaluated in the phase II single-arm DESTINY-Breast01 trial (55) enrolling patients pretreated with trastuzumab and T-DM1. Patients included in the study had received a median of six previous treatments, however achieved an impressive ORR of $60.9 \%$ (95\% CI: 53.4-68.0), with a median duration of response of 14.8 months (95\% CI: 13.8-16.9). As far as tolerability is concerned, among myelosuppression and nausea, interstitial lung disease occurred in $13.6 \%$ of the patients, including one death. Thus, careful monitoring for pulmonary symptoms is considered mandatory during treatment.

Interestingly, DESTINY-Breast01 included 24 patients with pretreated asymptomatic brain metastases. ORR was similar to the whole patient population $(58,3 \%, 95 \%$ CI $36,3-77.9)$, with a median duration of response of 16.9 months $(95 \% \mathrm{CI}$ : 5.7-16.9) (56). In fact, one patient experienced a $>50 \%$ regression of in-brain disease.

Antibody-drug conjugate trastuzumab duocarmazine (SYD985). Trastuzumab duocarmazine is composed of trastuzumab linked, via a cleavable linker, to a prodrug of the alkylating agent duocarmycin. Evaluation of the drug in a phase I dose-escalation and dose-expansion study (57) including patients with different solid tumors showed an ORR of $33 \%$ (16 of 48 patients) in HER2-overexpressing breast cancer and $29 \%$ (9 of 32 patients) in hormone-receptor positive, HER2-low breast cancer. A phase III trial comparing trastuzumab duocarmazine to physician's choice treatment in patients who have progressed after two anti-HER2 therapies is currently recruiting (NCT03262935).

Several other antibody-drug conjugates in earlier stages of development are being investigated in preclinical and clinical trials (A166, ALT-P7, ARX788, DHES0815A, MEDI4276, RC48, XMT-1522) (58).
HER2-targeting monoclonal antibody Margetuximab (MGAH22). Fc-receptor (FcR) polymorphism is considered one of the mechanisms of resistance to trastuzumab by reducing the trastuzumab-mediated cellular cytotoxicity. Margetuximab is a chimeric IgG monoclonal antibody with similar antiproliferative effects as trastuzumab, but greater affinity to variants of the CD16A receptor (59).

Margetuximab in later-line setting. Based on the rationale that increased affinity can enhance antibody-mediated immunity, margetuximab versus trastuzumab in heavily pretreated patients has been investigated in the phase III SOPHIA trial (60). Patients exposed to two or more HER2-targeting regimens were randomized to margetuximab plus chemotherapy or trastuzumab plus chemotherapy. PFS was significantly longer in the margetuximab arm in the ITT population (5.8 vs. 4.9 months, $\mathrm{HR}=0.76, \mathrm{P}=0.033)$ and, interestingly, in patients harboring the CD16A-158 F allele (6.9 vs. 5.1 months, $\mathrm{HR}=0.68, \mathrm{P}=0.005)$, which is associated with poor clinical response to trastuzumab. Furthermore, OS in the second interim analysis of patients carrying the CD16A-158F allele favored margetuximab, although not significantly (23.7 vs. 19.4 months, $\mathrm{P}=0.087$ ). Trastuzumab performed better in the homozygous patients, although clinical characteristics of the population were not balanced, with more patients with visceral and brain metastasis and older age being assigned to margetuximab. Final OS analysis is awaited in order to better define the role of margetuximab in this setting.

BispecificantibodyZW25.ZW25 is abispecific HER2-targeting antibody that simultaneously binds two non-overlapping HER2 epitopes, resulting in dual HER2 blockade with increased tumor cell binding and internalization (61). Effectiveness and safety of ZW25 in HER2-expressing tumors are being currently investigated in a 3-part study (NCT02892123). Part 1 has recently demonstrated clinical benefit in heavily pretreated patients with a variety of solid tumors, with a median PFS of 5.2 months and ORR $>30 \%$ (62), while no dose-limiting adverse events occurred. Parts 2 and 3 of the study evaluating ZW25 as monotherapy and combined with chemotherapy in specific tumor types are still ongoing. Furthermore, a currently recruiting phase II trial evaluates ZW25 in combination with palbociclib and fulvestrant in $\mathrm{HR}^{+} / \mathrm{HER} 2^{+}$breast cancer (NCT04224272).

Bispecific antibody MCLA-128. MCLA-128 is a bispecific antibody with high antibody-dependent cellular cytotoxicity binding HER2 and HER3. Preliminary clinical activity in solid tumors has been confirmed in a phase I trial (62), where, among 10 patients with heavily pretreated metastatic breast cancer, clinical benefit rate was $70 \%$ and the drug showed a favorable toxicity profile (only one infusion related reaction grade 4 in a total of 15 patients). An ongoing phase II trial investigates the addition of MCLA-128 to trastuzumab and vinorelbine in HER2 ${ }^{+}$tumors, as well as to endocrine therapy in $\mathrm{HR}^{+} / \mathrm{HER} 2$ low tumors (NCT03321981).

Cyclin dependent kinases (CDK)4/6 inhibitors. CDK4/6 inhibitors target the cell proliferative activity of CDK4/6 in the estrogen signaling pathway and are currently, in combi- 
nation with endocrine therapy, the standard-of-care in the treatment of hormone receptor positive/HER2 negative breast cancer (63). Potential for integration of CDK4/6 inhibitors in HER2-positive disease was first confirmed in the phase II monarchHER trial (64), in which patients were randomized to receive either abemaciclib, fulvestrant and trastuzumab $(\operatorname{arm} \mathrm{A})$, or abemaciclib and trastuzumab (arm B) or trastuzumab plus chemotherapy ( $\operatorname{arm} \mathrm{C}$ ). The triple combination significantly improved PFS (8.3 vs. 5.5 months, HR=0.67, $\mathrm{P}=0.051)$ compared to trastuzumab plus chemotherapy. On the other hand, no benefit was demonstrated for the abemaciclib/trastuzumab arm. According to an exploratory analysis, median overall survival is to date not significantly different between arms A and C (24 vs. 21 months).

$\mathrm{CDK} 4 / 6$ inhibition in $\mathrm{ER}^{+} / \mathrm{HER} 2^{+}$disease is being further studied in ongoing clinical trials. The phase II PATRICIA trial (65) evaluates the combination of palbociclib and trastuzumab in postmenopausal patients after 2-4 lines of HER2-directed treatments. During phase I of the trial, 60 patients were divided in 3 cohorts: ER-negative (cohort A), ER-positive (cohort B1) and ER-positive receiving letrozole (cohort B2). Phase I confirmed the efficacy of the regimen for both the luminal A and luminal B subtypes (median PFS 12.4 vs. 4.1 months in cohort $\mathrm{A}, \mathrm{p}=0.025$ ). Recruitment in phase II is ongoing with a target of a total of 232 patients (NCT02448420). In accordance with the known safety profile of CDK4/6 inhibitors, high grade neutropenia was observed in at least $80 \%$ of the participants. In the phase III PATINA trial (66), patients receive 6-8 cycles of chemotherapy with taxane or vinorelbine plus dual HER2-directed treatment with trastuzumab and pertuzumab and are then randomized to trastuzumab/pertuzumab and endocrine treatment with or without palbociclib. Primary endpoint of the study is PFS (NCT02947685).

PI3K/AKT/mTOR inhibition. A potential mechanism of resistance to HER2-targeted therapy is the permanent upregulation of the PI3K/AKT/mTOR pathway, often due to mutations of the PIK3CA gene or loss of function of the PTEN tumor suppressing phosphatase (67). Various studies have tested the hypothesis that combined targeting of PIK/AKT/mTOR and HER2 could overcome HER2 resistance.

Everolimus. Everolimus is an mTOR inhibitor that showed promising results in combination with trastuzumab and chemotherapy in early studies (68), nonetheless failed to demonstrate clinically significant benefit in the phase III BOLERO-3 (69) and BOLERO-1 (70) trials.

BOLERO-3 assessed the addition of everolimus to trastuzumab and chemotherapy in patients with disease progression under HER2-inhibition. Everolimus led to a median PFS of 7.0 vs. 5.8 months (HR=0.78, 95\% CI: 0.65-0.95, $\mathrm{P}=0.0067)$, a statistically significant but moderate benefit. In BOLERO-1, a study including patients in the first-line setting, hazard of progression was similar in both groups (15.0 vs. 14.5 months, $\mathrm{HR}=0.89$, 95\% CI: 0.73-1.08, $\mathrm{P}=0.1166$ ), while everolimus was associated with increased toxicity. However, according to a combined exploratory analysis of both studies, the subpopulation of patients with hyperactivation of the PI3K pathway seemed to profit from everolimus in terms of PFS (71).

\author{
$1^{\text {st }}$ line setting \\ Trastuzumab + Pertuzumab \pm Taxane \\ (mainstay) \\ Contraindication to chemotherapy: \\ Trastuzumab + Pertuzumab \\ Selected cases* of HR-positive disease: \\ $\mathrm{AI} \pm$ Trastuzumab or Lapatinib

\section{$2^{\text {nd }}$ line setting T-DM1} \\ Further line setting \\ If not previously received: T-DM1 \\ If not previously received: Pertuzumab in \\ combination with Trastuzumab \\ If T-DM1 / Pertuzumab previously \\ received multiple options available \\ (insufficient evidence to recommend one \\ regimen over another) \\ Trastuzumab + Chemotherapy \\ Lapatinib + Trastuzumab \\ Lapatinib + Capecitabine \\ Lapatinib + AI (HR-positive disease) \\ Preferred in CNS disease \\ Neratinib + Capecitabine \\ Tucatinib + Trastuzumab + Capecitabine \\ Pyrotinib + Capecitabine \\ Trastuzumab deruxtecan
}

Figure 1. Proposed treatment algorithm for patients with metastatic HER2-positive breast cancer. "Low burden disease, long disease-free interval and contraindication for HER2-targeted treatment. HR, hormone receptor; $\mathrm{AI}$, aromatase inhibitor; CNS, central nervous system.

PI3K inhibitors. Efficacy of PI3K blockage was initially tested using non-specific anti-PI3K drugs (68). A phase Ib trial evaluated treatment with the pan-PI3K inhibitor buparlisib and lapatinib in trastuzumab-pretreated patients (72). The combination led to a disease control rate of $79 \%$ (95\% CI: 57-92) and clinical benefit rate of $29 \%$ (95\% CI: 12-51) in a total of 24 patients, although high toxicity rates were found to be an important limiting factor.

More recently, newer agents managed to demonstrate clinical activity in small studies, while offering a better tolerated toxicity profile. For instance, the combination of alpelisib, a PI3K $\alpha$ isoform-specific inhibitor, and T-DM1 in trastuzumab-refractory disease resulted in an ORR of $43 \%$ in the total population $(n=17)$ and 30\% in T-DM1-resistant patients $(n=10)(73)$. Alpelisib combined with dual HER2-blockade is currently under evaluation as maintenance treatment in patients harboring a PIK3CA mutation in a phase III trial (NCT04208178). Other selective PI3K inhibitors such as taselisib and MEN1611 are also being tested in combination with anti-HER 2 treatment in early-phase trials (NCT02390427, NCT03767335) (68).

Immunotherapy. Although breast cancer is historically considered poorly immunogenic, HER2 ${ }^{+}$tumors have more 
Table I. Molecular targets of the various therapeutic agents in metastatic HER2-positive breast cancer

\begin{tabular}{ll}
\hline Drug Name(s) & \multicolumn{1}{c}{ Target } \\
\hline Anti-HER2 agents & \\
Trastuzumab, pertuzumab ${ }^{\mathrm{a}}$ & \\
Margentuximab $^{\mathrm{a}}$ & HER2 \\
ZW25 $^{\mathrm{b}}$ & HER2 (greater affinity to variants of the CD16A receptor) \\
MCLA-12 $^{\mathrm{b}}$ & Two non-overlapping HER2 epitopes \\
Lapatinib $^{\mathrm{c}}$ & HER2, HER3 \\
Neratinib, pyrotinib & \\
Tucatinib & EGFR, HER2 \\
Antibody-drug conjugates (trastuzumab + cytotoxic agent) & EGFR, HER2, HER3, HER4 \\
T-DM1 & HER2 \\
Trastuzumab deruxtecan & \\
Trastuzumab duocarmazine & HER2 + microtubules \\
CDK4/6 inhibitors (estrogen signaling pathway) & HER2 + topoisomerase 1 \\
Abemaciclib, palbociclib & HER2 + DNA (alkyl group binding) \\
PI3K/AKT/mTOR inhibitors & \\
Everolimus & CDK 4/6 \\
Buparlisib, alpelisib & \\
Immunotherapeutic agents & mTOR \\
Atezolizumab & PI3K \\
Pembrolizumab & \\
\hline
\end{tabular}

${ }^{a}$ Monoclonal antibodies. ${ }^{b}$ Bispecific antibodies. ${ }^{c}$ Tyrosine kinase inhibitors. EGFR, epidermal growth factor receptor; CDK, cyclin dependent kinase; mTOR, mechanistic target of rapamycin; PI3K, phosphatidylinositol-4,5-biphosphate 3-kinase; PD-1, programmed cell death protein 1; PD-L1, programmed cell death-ligand 1.

often higher expression of possible predictive biomarkers such as programmed death-ligand 1 (PD-L1), tumor-infiltrating lymphocytes (TILs) and tumor mutational burden (TMB) compared to luminal subtypes (74). Trastuzumab is also believed to enhance immunogenicity due to ADCC (59). Based on this rationale, immunotherapy with immune checkpoint-inhibitors has been evaluated in HER $2^{+}$breast cancer with considerable results in certain subgroups of patients.

Atezolizumab immunotherapy. Atezolizumab is a monoclonal IgG1 antibody directed against PD-L1. Efficacy in metastatic HER $2^{+}$breast cancer has been tested in the phase II KATE2 trial $(75,76)$, where patients with progressive disease after trastuzumab and taxane were randomized to receive T-DM1 with or without atezolizumab and were stratified in two cohorts based on PD-L1 expression. There was no statistical significance in PFS in the intention-to-treat population $(\mathrm{HR}=0.82$, $\mathrm{P}=0.3332$ ), but an exploratory analysis of the PD-L1-positive subgroup (PD-L1 $\geq 1 \%$ ) revealed a numerical PFS benefit in the atezolizumab arm (HR=0.60, 95\% CI: 0.32-1.11). 1-year OS also favored not significantly the atezolizumab group $(\mathrm{HR}=0.55$, 95\% CI: 0.22-1.38). However, generalizability of the results remains uncertain due to the limited number of patients $(n=19)$.

Pembrolizumab. Combination of pembrolizumab, a programmed cell death protein-1 (PD-1) inhibitor, with trastuzumab in patients pretreated with trastuzumab was evaluated in the phase Ib-II PANACEA trial (77). In PD-L1-positive tumors, ORR was $15 \%$ (6 of 40 patients) and disease control rate (DCR) $25 \%$, while there were no objective responders among PD-L1-negative patients. ORR reached 39\% and DCR $47 \%$ in a subgroup of PD-L1-positive patients with TILs $>5 \%$ in the metastatic lesion, further underlying the need for reliable biomarkers allowing efficient patient selection.

Anyhow, more data are necessary to define the role of immune checkpoint inhibitors in HER2 positive metastatic breast cancer. Several phase I and II trials evaluating immunotherapy with PD-1/PD-L1 and CTLA4-blockade in HER2 positive breast cancer are currently ongoing (78).

\section{Proposed treatment algorithm}

Based on the data presented above, a proposed treatment algorithm for patients with metastatic HER2-positve breast cancer is shown in (Fig. 1). Distinct molecular targets of the different drugs assessed in the treatment of these patients are presented in (Table I).

\section{Conclusion}

Prognosis of HER2-positive metastatic breast cancer has improved drastically since the introduction of HER2-targeted therapies. However, acquired resistance to treatment is common in the course of the disease, with the development of brain metastases remaining a difficult problem in clinical practice. Various novel agents are currently under investigation either as monotherapy or in combination with existing regimens with encouraging results in pretreated patients. Integration of new treatments for specific subgroups such as patients with HR-positive/HER2-positive tumors or CNS disease could 
further improve prognosis. Most importantly, identification of predictive factors is crucial to better determine the most appropriate therapeutic approach for different patients.

\section{Acknowledgements}

Not applicable.

\section{Funding}

No funding was received.

\section{Availability of data and materials}

Not applicable.

\section{Authors' contributions}

MM and IPT wrote the manuscript, GG, SI and NKS critically revised the manuscript and EAK revised the manuscript and supervised the work. All authors read and approved the final manuscript. MM and EAK confirm the authenticity of all the data.

\section{Ethics approval and consent to participate}

Not applicable.

\section{Patient consent for publication}

Not applicable.

\section{Competing interests}

The authors declare that they have no competing interests.

\section{References}

1. Torre LA, Islami F, Siegel RL, Ward EM and Jemal A: Global cancer in women: Burden and trends. Cancer Epidemiol Biomarkers Prev 26: 444-457, 2017.

2. Slamon DJ, Clark GM, Wong SG, Levin WJ, Ullrich A and McGuire WL: Human breast cancer: Correlation of relapse and survival with amplification of the HER-2/neu oncogene. Science 235: 177-182, 1987.

3. Burstein HJ: The distinctive nature of HER2-positive breast cancers. N Engl J Med 353: 1652-1654, 2005.

4. Kennecke H, Yerushalmi R, Woods R, Cheang MC, Voduc D, Speers $\mathrm{CH}$, Nielsen TO and Gelmon K: Metastatic behavior of breast cancer subtypes. J Clin Oncol 28: 3271-3277, 2010.

5. Harbeck N, Penault-Llorca F, Cortes J, Gnant M, Houssami N, Poortmans P, Ruddy K, Tsang J and Cardoso F: Breast cancer. Nat Rev Dis Primers 5: 66, 2019.

6. Iqbal $\mathrm{N}$ and Iqbal $\mathrm{N}$ : Human epidermal growth factor receptor 2 (HER2) in cancers: Overexpression and therapeutic implications. Mol Biol Int 2014: 852748, 2014.

7. Mendes D, Alves C, Afonso N, Cardoso F, Passos-Coelho JL, Costa L, Andrade S and Batel-Marques F: The benefit of HER2-targeted therapies on overall survival of patients with metastatic HER2-positive breast cancer - a systematic review. Breast Cancer Res 17: 140, 2015.

8. Hudis CA: Trastuzumab - mechanism of action and use in clinical practice. N Engl J Med 357: 39-51, 2007.

9. Vogel CL, Cobleigh MA, Tripathy D, Gutheil JC, Harris LN, Fehrenbacher L, Slamon DJ, Murphy M, Novotny WF, Burchmore M, et al: Efficacy and safety of trastuzumab as a single agent in first-line treatment of HER2-overexpressing metastatic breast cancer. J Clin Oncol 20: 719-726, 2002.
10. Slamon DJ, Leyland-Jones B, Shak S, Fuchs H, Paton V, Bajamonde A, Fleming T, Eiermann W, Wolter J, Pegram M, et al: Use of chemotherapy plus a monoclonal antibody against HER2 for metastatic breast cancer that overexpresses HER2. N Engl J Med 344: 783-792, 2001.

11. Andersson M, Lidbrink E, Bjerre K, Wist E, Enevoldsen K, Jensen AB, Karlsson P, Tange UB, Sørensen PG, Møller S, et al: Phase III randomized study comparing docetaxel plus trastuzumab with vinorelbine plus trastuzumab as first-line therapy of metastatic or locally advanced human epidermal grow th factor receptor 2-positive breast cancer: The HERNATA study. J Clin Oncol 29: 264-271, 2011.

12. Robert N, Leyland-Jones B, Asmar L, Belt R, Ilegbodu D, Loesch D, Raju R, Valentine E, Sayre R, Cobleigh M, et al: Randomized phase III study of trastuzumab, paclitaxel, and carboplatin compared with trastuzumab and paclitaxel in women with HER-2-overexpressing metastatic breast cancer. J Clin Oncol 24: 2786-2792, 2006.

13. Valero V, Forbes J, Pegram MD, Pienkowski T, Eiermann W, von Minckwitz G, Roche $\mathrm{H}$, Martin M, Crown J, Mackey JR, et al: Multicenter phase III randomized trial comparing docetaxel and trastuzumab with docetaxel, carboplatin, and trastuzumab as first-line chemotherapy for patients with HER2-gene-amplified metastatic breast cancer (BCIRG 007 study): Two highly active therapeutic regimens. J Clin Oncol 29: 149-156, 2011.

14. Baselga J, Manikhas A, Cortés J, Llombart A, Roman L, SemiglazovVF, Byakhov M, Lokanatha D, Forenza S, Goldfarb RH, et al: Phase III trial of nonpegylated liposomal doxorubicin in combination with trastuzumab and paclitaxel in HER2-positive metastatic breast cancer. Ann Oncol 25: 592-598, 2014.

15. Hamberg P, Bos MM, Braun HJ, Stouthard JM, van Deijk GA, Erdkamp FL, van der Stelt-Frissen IN, Bontenbal M, Creemers GJ, Portielje JE, et al; Dutch Breast Cancer Trialists' Group (BOOG): Randomized phase II study comparing efficacy and safety of combination-therapy trastuzumab and docetaxel vs. sequential therapy of trastuzumab followed by docetaxel alone at progression as first-line chemotherapy in patients with HER 2+ metastatic breast cancer: HERTAX trial. Clin Breast Cancer 11: 103-113, 2011.

16. Inoue K, Nakagami K, Mizutani M, Hozumi Y, Fujiwara Y, Masuda N, Tsukamoto F, Saito M, Miura S, Eguchi K, et al: Randomized phase III trial of trastuzumab monotherapy followed by trastuzumab plus docetaxel versus trastuzumab plus docetaxel as first-line therapy in patients with HER2-positive metastatic breast cancer: The JO17360 Trial Group. Breast Cancer Res Treat 119: 127-136, 2010.

17. Pagani O, Klingbiel D, Ruhstaller T, Nolè F, Eppenberger S, Oehlschlegel C, Bernhard J, Brauchli P, Hess D, Mamot C, et al; Swiss Group for Clinical Cancer Research (SAKK): Do all patients with advanced HER2 positive breast cancer need upfront-chemo when receiving trastuzumab? Randomized III trial SAKK 22/99. Ann Oncol 28: 305-312, 2017.

18. Baselga J, Cortés J, Kim SB, Im SA, Hegg R, Im YH, Roman L, Pedrini JL, Pienkowski T, Knott A, et al; CLEOPATRA Study Group: Pertuzumab plus trastuzumab plus docetaxel for metastatic breast cancer. N Engl J Med 366: 109-119, 2012.

19. Swain SM, Baselga J, Kim S-B, Ro J, Semiglazov V, Campone M, Ciruelos E, Ferrero JM, Schneeweiss A, Heeson S, et al; CLEOPATRA Study Group: Pertuzumab, trastuzumab, and docetaxel in HER2-positive metastatic breast cancer. N Engl J Med 372: 724-734, 2015.

20. Swain SM, Miles D, Kim SB, Im YH, Im SA, Semiglazov V, Ciruelos E, Schneeweiss A, Loi S, Monturus E, et al: Pertuzumab, trastuzumab, and docetaxel for HER2-positive metastatic breast cancer (CLEOPATRA): end-of-study results from a double-blind, randomised, placebo-controlled, phase 3 study. Lancet Oncol 21: 519-530, 2020.

21. Miles D, Im YH, Fung A, Yoo B, Knott A, Heeson S, Beattie MS and Swain SM: Effect of docetaxel duration on clinical outcomes: Exploratory analysis of CLEOPATRA, a phase III randomized controlled trial. Ann Oncol 28: 2761-2767, 2017.

22. Bachelot T, Ciruelos E, Schneeweiss A, Puglisi F, Peretz-Yablonski T, Bondarenko I, Paluch-Shimon S, Wardley A, Merot JL, du Toit Y, et al; PERUSE investigators: Preliminary safety and efficacy of first-line pertuzumab combined with trastuzumab and taxane therapy for HER2-positive locally recurrent or metastatic breast cancer (PERUSE). Ann Oncol 30: 766-773, 2019. 
23. Perez EA, López-Vega JM, Petit T, Zamagni C, Easton V, Kamber J, Restuccia E and Andersson M: Safety and efficacy of vinorelbine in combination with pertuzumab and trastuzumab for first-line treatment of patients with HER2-positive locally advanced or metastatic breast cancer: VELVET Cohort 1 final results. Breast Cancer Res 18: 126, 2016.

24. Inoue K, Ninomiya J, Saito T, Okubo K, Nakakuma T, Yamada H, Kimizuka K and Higuchi T; SBCCSG-36 investigators: Eribulin, trastuzumab, and pertuzumab as first-line therapy for patients with HER2-positive metastatic breast cancer: A phase II, multicenter, collaborative, open-label, single-arm clinical trial. Invest New Drugs 37: 538-547, 2019. Erratum in: Invest New Drugs 37: 592-593, 2019.

25. Urruticoechea A, Rizwanullah M, Im SA, Ruiz ACS, Láng I, Tomasello G, Douthwaite H, Badovinac Crnjevic T, Heeson S, Eng-Wong J, et al: Randomized phase III trial of trastuzumab plus capecitabine with or without pertuzumab in patients with human epidermal growth factor receptor 2-positive metastatic breast cancer who experienced disease progression during or after trastuzumab-based therapy. J Clin Oncol 35: 3030-3038, 2017.

26. Urruticoechea A, Rizwanullah M, Im SA, Sánchez Ruiz AC, Lang I, Tomasello G, Douthwaite H, Badovinac Crnjevic T, Heeson S, Eng-Wong J, et al: Final overall survival (OS) analysis of PHEREXA: A randomized phase III trial of trastuzumab $(\mathrm{H})+$ capecitabine $(\mathrm{X}) \pm$ pertuzumab $(\mathrm{P})$ in patients with HER2-positive metastatic breast cancer (MBC) who experienced disease progression during or after $\mathrm{H}$-based therapy. J Clin Oncol 36 (Suppl 15): 1013, 2018.

27. Lewis Phillips GD, Li G, Dugger DL, Crocker LM, Parsons KL, Mai E, Blättler WA, Lambert JM, Chari RV, Lutz RJ, et al: Targeting HER2-positive breast cancer with trastuzumab-DM1, an antibody-cytotoxic drug conjugate. Cancer Res 68: 9280-9290, 2008

28. Krop IE, Kim SB, González-Martín A, LoRusso PM, Ferrero JM, Smitt M, Yu R, Leung AC and Wildiers H; TH3RESA study collaborators: Trastuzumab emtansine versus treatment of physician's choice for pretreated HER2-positive advanced breast cancer (TH3RESA): A randomised, open-label, phase 3 trial. Lancet Oncol 15: 689-699, 2014

29. Krop IE, Kim SB, Martin AG, LoRusso PM, Ferrero JM, Badovinac-Crnjevic T, Hoersch S, Smitt M and Wildiers $H$ Trastuzumab emtansine versus treatment of physician's choice in patients with previously treated HER2-positive metastatic breast cancer(TH3RESA): Final overall survival results from a randomised open-label phase 3 trial. Lancet Oncol 18: 743-754, 2017.

30. Verma S, Miles D, Gianni L, Krop IE, Welslau M, Baselga J, Pegram M, Oh DY, Diéras V, Guardino E, et al; EMILIA Study Group: Trastuzumab emtansine for HER2-positive advanced breast cancer. N Engl J Med 367: 1783-1791, 2012

31. Diéras V, Miles D, Verma S, Pegram M, Welslau M, Baselga J, Krop IE, Blackwell K, Hoersch S, Xu J, et al: Trastuzumab emtansine versus capecitabine plus lapatinib in patients with previously treated HER2-positive advanced breast cancer (EMILIA): A descriptive analysis of final overall survival results from a randomised, open-label, phase 3 trial. Lancet Oncol 18: 732-742, 2017.

32. Perez EA, Barrios C, Eiermann W, Toi M, Im YH, Conte P, Martin M, Pienkowski T, Pivot X, Burris H III, et al: Trastuzumab emtansine with or without pertuzumab versus trastuzumab plus taxane for human epidermal growth factor receptor 2-positive, advanced breast cancer: Primary results From the Phase III MARIANNE Study. J Clin Oncol 35: 141-148, 2017. Erratum in: J Clin Oncol 35: 2342, 2017.

33. Perez EA, Barrios C, Eiermann W, Toi M, Im YH, Conte P, Martin M, Pienkowski T, Pivot XB, Burris HA III, et al: Trastuzumab emtansine with or without pertuzumab versus trastuzumab with taxane for human epidermal growth factor receptor 2-positive advanced breast cancer: Final results from MARIANNE. Cancer 125: 3974-3984, 2019.

34. Roskoski R Jr: Small molecule inhibitors targeting the EGFR/ErbB family of protein-tyrosine kinases in human cancers. Pharmacol Res 139: 395-411, 2019.

35. Cameron D, Casey M, Press M, Lindquist D, Pienkowski T, Romieu CG, Chan S, Jagiello-Gruszfeld A, Kaufman B, Crown J, et al: A phase III randomized comparison of lapatinib plus capecitabine versus capecitabine alone in women with advanced breast cancer that has progressed on trastuzumab: Updated efficacy and biomarker analyses. Breast Cancer Res Treat 112: 533-543, 2008
36. Gelmon KA, Boyle FM, Kaufman B, Huntsman DG, Manikhas A, Di Leo A, Martin M, Schwartzberg LS, Lemieux J, Aparicio S, et al: Lapatinib or trastuzumab plus taxane therapy for human epidermal growth factor receptor 2-positive advanced breast cancer: Final results of NCIC CTG MA.31. J Clin Oncol 33: 1574-1583, 2015.

37. Blackwell KL, Burstein HJ, Storniolo AM, Rugo HS, Sledge G, Aktan G, Ellis C, Florance A, Vukelja S, Bischoff J, et al: Overall survival benefit with lapatinib in combination with trastuzumab for patients with human epidermal growth factor receptor 2-positive metastatic breast cancer: Final results from the EGF104900 Study. J Clin Oncol 30: 2585-2592, 2012.

38. Prat A and Baselga J: The role of hormonal therapy in the management of hormonal-receptor-positive breast cancer with co-expression of HER2. Nat Clin Pract Oncol 5: 531-542, 2008.

39. Montagna E and Colleoni M: Hormonal treatment combined with targeted therapies in endocrine-responsive and HER2-positive metastatic breast cancer. Ther Adv Med Oncol 11: $1758835919894105,2019$.

40. Kaufman B, Mackey JR, Clemens MR, Bapsy PP, Vaid A, Wardley A, Tjulandin S, Jahn M, Lehle M, Feyereislova A, et al: Trastuzumab plus anastrozole versus anastrozole alone for the treatment of postmenopausal women with human epidermal growth factor receptor 2-positive, hormone receptor-positive metastatic breast cancer: Results from the randomized phase III TAnDEM study. J Clin Oncol 27: 5529-5537, 2009.

41. Johnston S, Pippen J Jr, Pivot X, Lichinitser M, Sadeghi S, Dieras V, Gomez HL, Romieu G, Manikhas A, Kennedy MJ, et al: Lapatinib combined with letrozole versus letrozole and placebo as first-line therapy for postmenopausal hormone receptor-positive metastatic breast cancer. J Clin Oncol 27: 5538-5546, 2009.

42. Huober J, Fasching PA, Barsoum M, Petruzelka L, Wallwiener D, Thomssen C, Reimer T, Paepke S, Azim HA, Ragosch V, et al: Higher efficacy of letrozole in combination with trastuzumab compared to letrozole monotherapy as first-line treatment in patients with HER2-positive, hormone-receptor-positive metastatic breast cancer - results of the eLEcTRA trial. Breast 21: 27-33, 2012

43. Rimawi M, Ferrero J-M, de la Haba-Rodriguez J, Poole C, De Placido S, Osborne CK, Hegg R, Easton V, Wohlfarth C and Arpino G; PERTAIN Study Group: First-line trastuzumab plus an aromatase inhibitor, with or without pertuzumab, in human epidermal growth factor receptor 2-positive and hormone receptor-positive metastatic or locally advanced breast cancer (PERTAIN): A randomized, open-label phase II trial. J Clin Oncol 36: 2826-2835, 2018.

44. Johnston SRD, Hegg R, Im SA, Park IH, Burdaeva O, Kurteva G, Press MF, Tjulandin S, Iwata H, Simon SD, et al: Phase III, randomized study of dual human epidermal grow th factor receptor 2 (HER2) blockade with lapatinib plus trastuzumab in combination with an aromatase inhibitor in postmenopausal women with HER2-positive, hormone receptor-positive metastatic breast cancer: ALTERNATIVE. J Clin Oncol 36: 741-748, 2018. Retraction in: J Clin Oncol 39: 95, 2021.

45. Saura C, Oliveira M, Feng Y, Dai M-S, Hurvitz SA, Kim SB, Moy B, Delaloge S, Gradishar WJ, Masuda N, et al: Neratinib plus capecitabine versus lapatinib plus capecitabine in HER2-positive metastatic breast cancer previously treated with $\geq 2$ HER2-directed regimens: Phase III NALA trial. J Clin Oncol 38: 3138-3149, 2019.

46. Awada A, Colomer R, Inoue K, Bondarenko I, Badwe RA, Demetriou G, Lee SC, Mehta AO, Kim SB, Bachelot T, et al: Neratinib plus paclitaxel vs trastuzumab plus paclitaxel in previously untreated metastatic ERBB2-positive breast cancer. The NEfERT-T randomized clinical trial. JAMA Oncol 2: 1557-1564, 2016.

47. Freedman RA, Gelman RS, Wefel JS, Melisko ME, Hess KR, Connolly RM, Van Poznak CH, Niravath PA, Puhalla SL, Ibrahim N, et al: Translational Breast Cancer Research Consortium (TBCRC) 022: A phase II trial of neratinib for patients with human epidermal growth factor receptor 2-positive breast cancer and brain metastases. J Clin Oncol 34: 945-952, 2016.

48. Freedman RA, Gelman RS, Anders CK, Melisko ME, Parsons HA, Cropp AM, Silvestri K, Cotter CM, Componeschi KP, Marte JM, et al; Translational Breast Cancer Research Consortium: TBCRC 022: A phase II trial of neratinib and capecitabine for patients with human epidermal growth factor receptor 2-positive breast cancer and brain metastases. J Clin Oncol 37: 1081-1089, 2019. 
49. Ma F, Ouyang Q, Li W, Jiang Z, Tong Z, Liu Y, Li H, Yu S, Feng J, Wang $S$, et al: Pyrotinib or lapatinib combined with capecitabine in HER2-positive metastatic breast cancer with prior taxanes, anthracyclines, and/or trastuzumab: A randomized, phase II study. J Clin Oncol 37: 2610-2619, 2019.

50. Xu B, Yan M, Ma F, Hu XC, Feng FJ, Ouyang Q, Tong Z, Li H, Zhang Q, Sun T, et al. Pyrotinib or lapatinib plus capecitabine for HER2+ metastatic breast cancer (PHOEBE): a randomized phase III trial. J Clin Oncol 38 (Suppl 15): 1003, 2020.

51. Jiang Z, Yan M, Hu X, Zhang Q, Ouyang Q, Feng J, Yin Y, Sun T, Tong Z, Wang X, et al: Pyrotinib combined with capecitabine in women with HER 2+ metastatic breast cancer previously treated with trastuzumab and taxanes: A randomized phase III study. J Clin Oncol 37 (Suppl 15): 1001, 2019.

52. Blair HA: Pyrotinib: First global approval. Drugs 78: 1751-1755, 2018.

53. Murthy RK, Loi S, Okines A, Paplomata E, Hamilton E, Hurvitz SA, Lin NU, Borges V, Abramson V, Anders C, et al: Tucatinib, trastuzumab and capecitabine for HER2-positive metastatic breast cancer. N Engl J Med 382: 597-609, 2020.

54. Lin NU, Borges V, Anders C, Murthy RK, Paplomata E, Hamilton E, Hurvitz S, Loi S, Okines A, Abramson V, et al: Intracranial efficacy and survival with tucatinib plus trastuzumab and capecitabine for previously treated HER2-positive breast cancer with brain metastases in the HER2CLIMB trial. J Clin Oncol 38: 2610-2619, 2020.

55. Modi S, Saura C, Yamashita T, Park YH, Kim SB, Tamura K, Andre F, Iwata H, Ito Y, Tsurutani J, et al; DESTINY-Breast01 Investigators: Trastuzumab Deruxtecan in previously treated HER2-positive breast cancer. N Engl J Med 382: 610-621, 2020.

56. Jerusalem G, Park YH, Yamashita T, Hurvitz SA, Chen S, Cathcart J, Lee C and Perrin C: CNS metastases in HER2-positive metastatic breast cancer treated with trastuzumab deruxtecan: DESTINY-Breast01 subgroup analyses. Ann Oncol 31: Abstract nr. 1380, 2020

57. Banerji U, van Herpen CML, Saura C, Thistlethwaite F, Lord S, Moreno V, Macpherson IR, Boni V, Rolfo C, de Vries EGE, et al: Trastuzumab duocarmazine in locally advanced and metastatic solid tumours and HER2-expressing breast cancer: A phase 1 dose-escalation and dose-expansion study. Lancet Oncol 20 . 1124-1135, 2019

58. Rinnerthaler G, Gampenrieder SP and Greil R: HER2 directed antibody-drug-conjugates beyond T-DM1 in breast cancer. Int J Mol Sci 20: 115, 2019

59. Dolcetti R: Predictive value of FcR polymorphisms. A further step on the long and winding road to application. JAMA Oncol 3: 342-343, 2017

60. Rugo HS, Im SA, Cardoso F, Cortes J, Curigliano G, Pegram MD, Musolino A, Bachelot T, Wright GS, De Laurentiis M, et al: Phase III SOPHIA study of margetuximab + chemotherapy vs trastuzumab + chemotherapy in patients with HER $2+$ metastatic breast cancer after prior anti-HER2 therapies: second interim overall survival analysis. Cancer Res 80: Abstract Nr. GS1-02, 2019

61. Meric-Bernstam F, Hanna D, Beeram M, Lee K, Kang Y, Chaves J, Lee J, Goodwin R, Vaklavas C, Oh D, et al: Safety, anti-tumor activity, and biomarker results of the HER2-targeted bispecific antibody ZW25 in HER2-expressing solid tumors. Ann Oncol 30 (Suppl 5): v159-v193, 2019.

62. Alsina M, Boni V, Schellens J, Moreno V, Bol K, Westendorp M, Sirulnik LA, Tabernero J and Calvo E: First-in-human phase 1/2 study of MCLA-128, a full length IgG1 bispecific antibody targeting HER2 and HER3: Final phase 1 data and preliminary activity in HER2+ metastatic breast cancer (MBC). J Clin Oncol 35 (Suppl 15): 2522, 2017

63. Schettini F, Giudici F, Giuliano M, Cristofanilli M, Arpino G Del Mastro L, Puglisi F, De Placido S, Paris I, De Placido P, et al: Overal survival of CDK4/6-inhibitors-based treatments in clinically relevant subgroups of metastatic breast cancer: Systematic review and meta-analysis. J Natl Cancer Inst 112: 1089-1097, 2020.

64. Tolaney SM, Wardley AM, Zambelli S, Hilton JF, Troso-Sandoval TA, Ricci F, Im SA, Kim SB, Johnston SR, Chan A, et al: Abemaciclib plus trastuzumab with or without fulvestrant versus trastuzumab plus standard-of-care chemotherapy in women with hormone receptor-positive, HER2-positive advanced breast cancer (monarcHER): A randomised, open-label, phase 2 trial. Lancet Oncol 21: 763-775, 2020.
65. Ciruelos E, Villagrasa P, Paré L, Oliveira M, Pernas S, Cortés J, Soberino J, Adamo B, Vazquez S, Martínez N, et al: SOLTI-1303 PATRICIA phase II trial (STAGE 1) - Palbociclib and trastuzumab in postmenopausal patients with HER2-positive metastatic breast cancer. Cancer Res 79 (Suppl 4): Abstract nr PD3-03, 2019.

66. Metzger O, Mandrekar S, Loibl S, Mundhenke C, Seiler S, Valagussa P, Lim E, Tripathy D, Winer EP, Huang C, et al: PATINA: A randomized, open label, phase III trial to evaluate the efficacy and safety of palbociclib + anti-HER2 therapy + endocrine therapy (ET) vs. anti-HER2 therapy + ET after induction treatment for hormone receptor positive (HR+)/HER2-positive metastatic breast cancer (MBC). Cancer Res 79 (Suppl 4): Abstract nr OT3-02-07, 2019.

67. Wang Q, Liu P, Spangle JM, Von T, Roberts TM, Lin NU, Krop IE, Winer EP and Zhao JJ: PI3K-p110 $\alpha$ mediates resistance to HER2-targeted therapy in HER2+, PTEN-deficient breast cancers. Oncogene 35: 3607-3612, 2016.

68. Mezni E, Vicier C, Guerin M, Sabatier R, Bertucci F and Gonçalves A: New therapeutics in HER2-positive advanced breast cancer: Towards a change in clinical practices? Cancers (Basel) 12: 1573, 2020.

69. André F, O'Regan R, Ozguroglu M, Toi M, Xu B, Jerusalem G, Masuda N, Wilks S, Arena F, Isaacs C, et al: Everolimus for women with trastuzumab-resistant, HER2-positive, advanced breast cancer (BOLERO-3): A randomised, double-blind, placebo-controlled phase 3 trial. Lancet Oncol 15: 580-591, 2014.

70. Hurvitz SA, André F, Jiang Z, Shao Z, Mano MS, Neciosup SP, Tseng LM, Zhang Q, Shen K, Liu D, et al: Combination of everolimus with trastuzumab plus paclitaxel as first-line treatment for patients with HER2-positive advanced breast cancer (BOLERO-1): A phase 3, randomised, double-blind, multicentre trial. Lancet Oncol 16: 816-829, 2015.

71. André F, Hurvitz S, Fasolo A, Tseng LM, Jerusalem G, Wilks S, O'Regan R, Isaacs C, Toi M, Burris H, et al: Molecular alterations and everolimus efficacy in human epidermal growth factor receptor 2-overexpressing metastatic breast cancers: Combined exploratory biomarker analysis From BOLERO-1 and BOLERO-3. J Clin Oncol 34: 2115-2124, 2016. Erratum in: J Clin Oncol 37: 357, 2019.

72. Guerin M, Rezai K, Isambert N, Campone M, Autret A, Pakradouni J, Provansal M, Camerlo J, Sabatier R, Bertucci F, et al: PIKHER2: A phase IB study evaluating buparlisib in combination with lapatinib in trastuzumab-resistant HER2-positive advanced breast cancer. Eur J Cancer 86: 28-36, 2017.

73. Jain S, Shah AN, Santa-Maria CA, Siziopikou K, Rademaker A, Helenowski I, Cristofanilli M and Gradishar WJ: Phase I study of alpelisib (BYL-719) and trastuzumab emtansine (T-DM1) in HER2-positive metastatic breast cancer (MBC) after trastuzumab and taxane therapy. Breast Cancer Res Treat 171: 371-381, 2018.

74. Viale G, Morganti S, Ferraro E, Zagami P, Marra A and Curigliano G: What therapies are on the horizon for HER2 positive breast cancer? Expert Rev Anticancer Ther 19: 811-822, 2019.

75. Emens LA, Esteva F, Beresford M, Saura C, De Laurentiis M, Kim SB, Im SA, Patre M, Wang Y, Mani A, et al: Results from KATE2, a randomized phase 2 study of atezolizumab (atezo)+trastuzumab emtasine (T-DM1) vs placebo (pbo)+T-DM1 in previously treated HER2+ advanced breast cancer (BC). Cancer Res 79 (Suppl 4): Abstract nr PD3-01, 2019.

76. Emens L, Esteva F, Beresford M, Saura C, De Laurentiis M, Kim SB, Im SA, Wang Y, Mani A, Shah J, et al: Overall survival (OS) in KATE2, a phase 2 study of programmed death ligand 1 (PD-L1) inhibitor atezolizumab (atezo)+trastuzumab emtasine (T-DM1) vs placebo (pbo)+T-DM1 in previously treated HER2+ advanced breast cancer (BC). Ann Oncol 30 (Suppl 5): v104-v142, 2019.

77. Loi S, Giobbie-Hurder A, Gombos A, Bachelot T, Hui R, Curigliano G, Campone M, Biganzoli L, Bonnefoi $\mathrm{H}$, Jerusalem G, et al; International Breast Cancer Study Group and the Breast International Group: Pembrolizumab plus trastuzumab in trastuzumab-resistant, advanced, HER2-positive breast cancer (PANACEA): A single-arm, multicentre, phase 1b-2 trial. Lancet Oncol 20: 371-382, 2019.

78. Ayoub NM, Al-Shami KM and Yaghan RJ: Immunotherapy for HER2-positive breast cancer: Recent advances and combination therapeutic approaches. Breast Cancer (Dove Med Press) 11: 53-69, 2019. 